\title{
Anisotropy Photoinduction during the Mass Associations of Dye Molecules in Gelatin Films
}

\author{
Tariel Ebralidze, Nadia Ebralidze, and Giorgi Mumladze \\ Department of Coherent Optics and Electronics, Vladimir Chavchanidze Institute of Cybernetics of the Georgian Technical University, \\ Sandro Euli Street 5, 0186 Tbilisi, Georgia
}

Correspondence should be addressed to Tariel Ebralidze; tarebra@yahoo.com

Received 7 December 2012; Revised 14 January 2013; Accepted 28 January 2013

Academic Editor: Rodrigo Martins

Copyright (C) 2013 Tariel Ebralidze et al. This is an open access article distributed under the Creative Commons Attribution License, which permits unrestricted use, distribution, and reproduction in any medium, provided the original work is properly cited.

By using the video microscopy, the picture of the formation of anisotropy photoinduction in the form of grains in time is shown, when anisotropy in the film is induced in individual micrograins and the concentration and sizes of the grains are modulated depending on the light exposition. This phenomenon was observed in the gelatin film dyed with the mixture prepared with the saturated solutions of Chrysophenine and Acridine Yellow mixed with 1:1 proportion.

\section{Introduction}

Gelatin films and films of other gels dyed with azo-dyes are worthy of attention in respect of their use in the optical use of data, nonlinear optics, holography and other fields. In the scientists' opinion, the anisotropy photoinduction in these films is the result of trans-cis-trans transformations by light $[1,2]$. However, to obtain high effect during the anisotropy photoinduction, the concentration of dyes in the film must be as high as possible. As for the increased concentration of dye in the solutions, it leads to the formation of molecule associations in a solid state playing an important role in the photo-induced effects [3-7].

Based on the study of the dependence of the spectral transmission in the crossed polarizers of the gelatin film dyed with Chrysophenine with photo-induced anisotropy on the active light exposition [3], an opinion about the possible anisotropy photoinduction in organic compounds in the form of grains and increase of concentration of grains and their areas particularly depending on the light exposition in the film was expressed.

The given work for the first time presents the visual evidence of the anisotropy photoinduction of the given kind in organic compound. The effect was observed in the gelatin film containing mass associations of the molecules obtained by mixing the saturated water solutions of Chrysophenine and Acridine Yellow with 1:1 proportion in gelatin [4].

\section{Experiment}

First, the saturated water solutions of Chrysophenine and Acridine Yellow were prepared for the experiment (see Figure 1).

Then, they were mixed with the proportion $1: 1$, and we shot the absorption spectra of the mixture and constituent components as 1, 2, and 3, respectively (see Figure 2). The spectra were shot with the Avantes spectroscope. Curve 4 in Figure 2 is the curve of the mixture deflection from additivity. It was obtained by subtracting the sum of the absorption spectra 2 and 3 of the mixture constituent components from mixture absorption spectrum 1 . As we can see, it shows the deflection from additivity, which evidences that the associations are made of different kinds of molecules.

During the experiment, we added the mixture of the saturated water solutions of Chrysophenine and Acridine Yellow dyes mixed with 1:1 proportion to $3 \%$ of gelatin. We poured the obtained solution on the microscope slide and dried it under room conditions. As a result, we obtained a thin (approximately 200 micron thick) film containing the molecule associations. We placed the film on the rotating table of the microscope fluorometer (Opton) and studied its image in terms of crossed polarizers. There are mass anisotropic associations observed in the film. Their images are seen as light microareas on the darkened background. At turning the microscope table, the light microareas in 


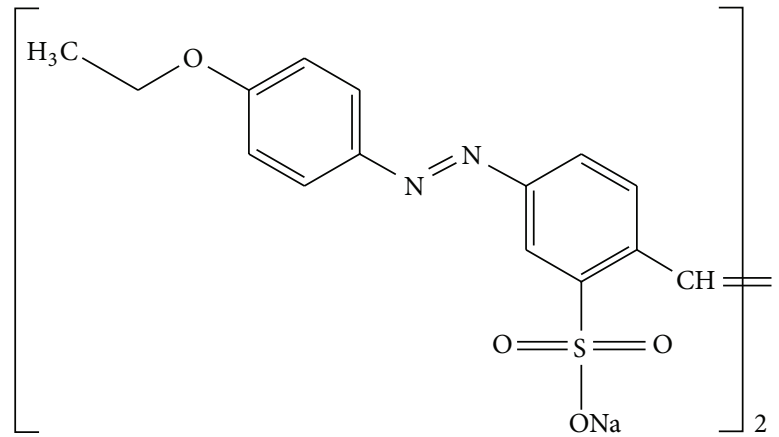

(a)<smiles>Cc1cc2cc3cc(C)c(N)cc3nc2cc1N</smiles>

(b)

Figure 1: Chemical structures of (a) Chrysophenine and (b) Acridine Yellow.

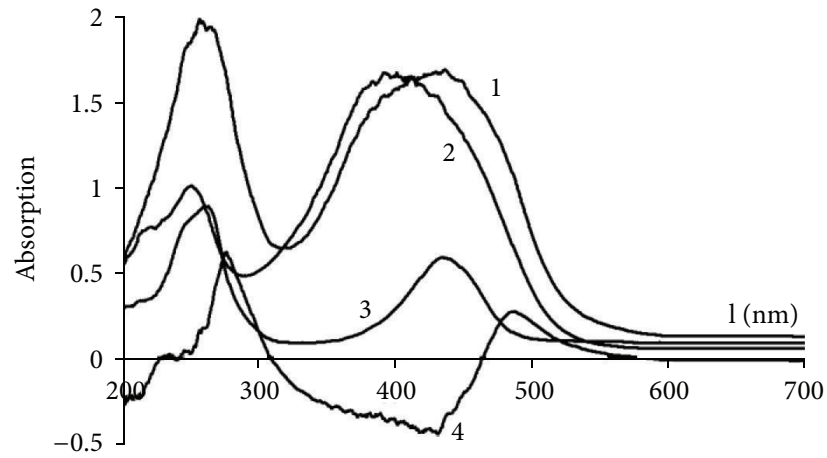

FIGURE 2: The absorption spectra of the mixture of the saturated water solutions of dyes Chrysophenine and Acridine Yellow mixed with 1: 1 proportion, their constituent components and the one showing the deflection from additivity as curves $1,2,3$, and 4 , respectively.

the film start to darken, and the new microareas start to illuminate on other sites instead. This means that there are anisotropic associations in the film with their optical axes being chaotically oriented generally. The associations with their optical axes making an approximately $45^{\circ}$ angle with the optical axes of the polarizer and analyzer in the given state are maximally lit, while the ones with their optical axes almost coinciding or making a $90^{\circ}$ angle with the optical axes of the polarizer and analyzer are darkened.

By irradiating the film with a narrow spectral band containing a $440 \mathrm{~nm}$ wavelength of a mercury lamp light, we arrived at the photodestruction of the molecule associations. The irradiated area was totally darkened during the experiment with the crossed polarizers.

By using video microscopy, we investigated the mode of formation of the picture of anisotropy in the time of exposition. Below, we give the photos made at successive time moments with certain intervals.

An image of the photo-induced anisotropy area in the crossed polarizers was shot with a 2-second interval in an anisotropy photoinduction dynamic mode on a microscopefluorometer slide with a microscope ocular camera in the automated shooting mode. Up to 200 figures were shot. The images in Figures 3(a), 3(b), 3(c), and 3(d) were selected out of those figures. They are shot at the moments with certain time intervals.
The darkened circle in Figure 3(a) is the site with isotropy obtained by the light irradiation. As it can be seen, no anisotropic grains are observed on the darkened site. From the beginning, they were there in the same way, as beyond this area. They were subject to photo-destruction by the action of nonpolarized light. Figure 3(b) shows the germs of the photoinduced anisotropic grains. This figure was selected some time from the start of the light irradiation.

The sizes and number of the photo-induced grains in Figure 3(c) are significantly increased. A photo-induced anisotropic image with its anisotropic areas so much increased as being merged with one another is given in Figure 3(d). This means that at the beginning of the active light exposition in the given film the anisotropy was induced in the individual microareas and not in all the exposed area. Then, the anisotropic microareas and their areas in particular increased depending on the light exposition.

\section{Conclusion}

Thus, by mixing the saturated solutions of Chrysophenine and Acridine Yellow with 1:1 proportion in gelatin, pouring it on the glass and drying under the room conditions, an approximately 200 -micron-thick film was obtained with the mass induction of different kinds of molecule associations in 


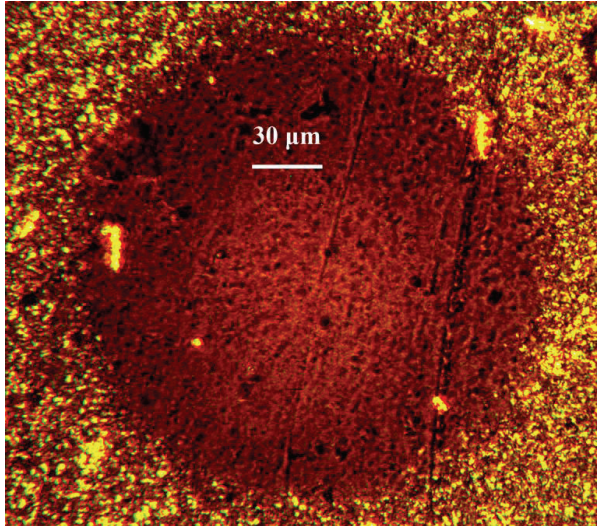

(a)

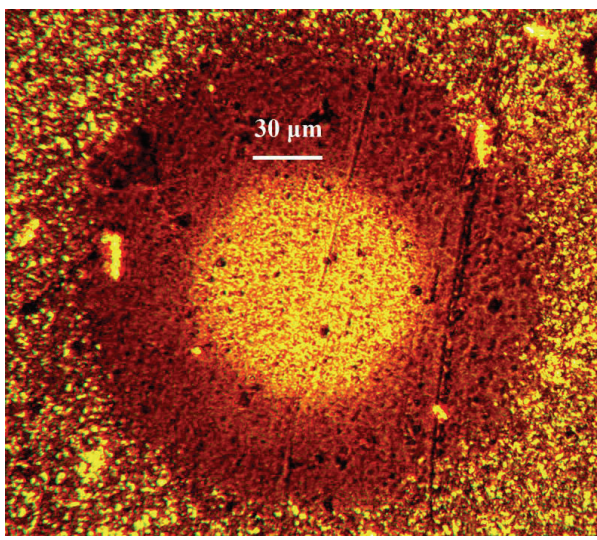

(c)

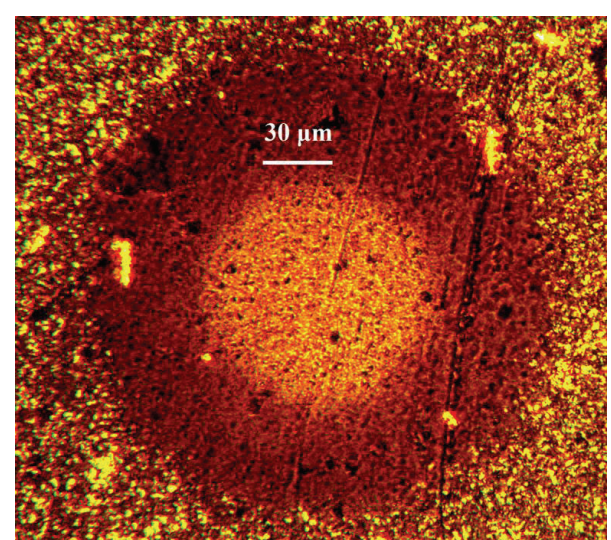

(b)

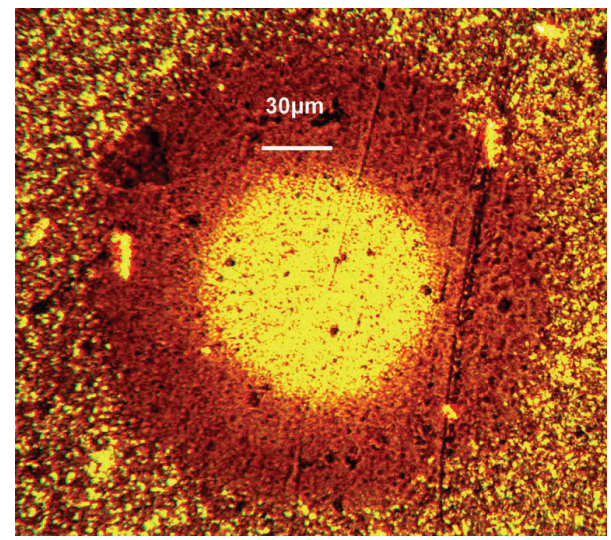

(d)

FIgURE 3: (a) The image of film obtained by irradiation with nonpolarized light. (b) The germs of the photo-induced anisotropic grains, selected some time from the start of the light irradiation. (c) The image of photoinduced anisotropic grains in the middle of exposition. (d) The image of photoinduced anisotropic grains at the end of exposition.

it. Under the action of the light from a narrow nonpolarized spectral band containing a $440 \mathrm{~nm}$ wavelength on the film, the irradiated area becomes isotropic; and by reacting with the linearly polarized light on it, the induction of the grains with their optical axes with the same orientation is obtained with their concentration and sizes being a light exposition function.

\section{Acknowledgment}

Investigations have been fulfilled by the financial support of Georgia's Shota Rustaveli National Science FoundationProject no. GNSF/ST08/1-365.

\section{References}

[1] A. M. Makushenko, B. S. Neporent, B. S. Neporent, and O. V. Stolbova, "Reverssible orientational photodichroism and photoisomerization of aromatic azo compounds. I," Optics and Spectroscopy (USSR), vol. 31, pp. 557-564, 1971.

[2] F. L. Labarthet, S. Freiberg, C. Pellerin, M. Pézolet, A. Natansohn, and P. Rochon, "Spectroscopic and optical characterization of a series of azobenzene-containing side-chain liquid crystalline polymers," Macromolecules, vol. 33, no. 18, pp. 6815$6823,2000$.

[3] T. D. Ebralidze and A. N. Mumladze, "Light-induced anisotropy in azo-dye-colored materials," Applied Optics, vol. 29, no. 4, pp. 446-447, 1990.

[4] T. D. Ebralidze, N. A. Ebralidze, G. A. Mumladze, and E. S. Kitsmarishvili, "Light-controlled mass formation of aggregates of molecules in organic compounds," Chinese Optics Letters, vol. 7, no. 9, pp. 823-825, 2009.

[5] D. Voloschenko and O. D. Lavrentovich, "Light-induced director-controlled microassembly of dye molecules from a liquid crystal matrix," Journal of Applied Physics, vol. 86, no. 9, pp. 4843-4846, 1999.

[6] B. J. Kim, S. Y. Park, and D. H. Choi, "Effect of molecular aggregation on the photo-induced anisotropy in amorphous polymethacrylate bearing an aminonitroazobenzene moiety," Bulletin of the Korean Chemical Society, vol. 22, no. 3, pp. 271275, 2001.

[7] A. Priimagi, A. Shevchenko, M. Kaivola, F. J. Rodriguez, M. Kauranen, and P. Rochon, "High and stable photoinduced anisotropy in guest-host polymer mediated by chromophore aggregation," Optics Letters, vol. 35, no. 11, pp. 1813-1815, 2010. 

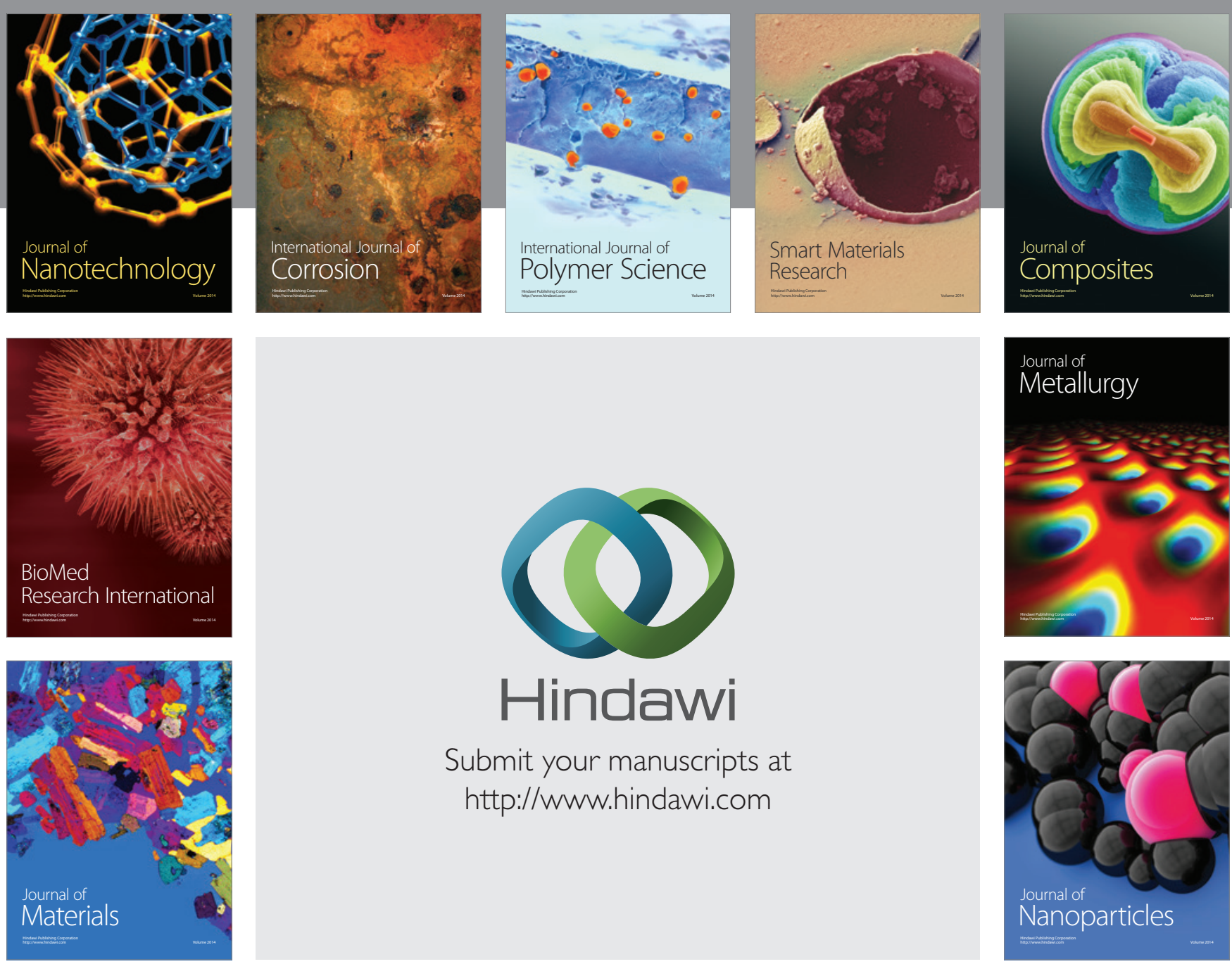

Submit your manuscripts at http://www.hindawi.com
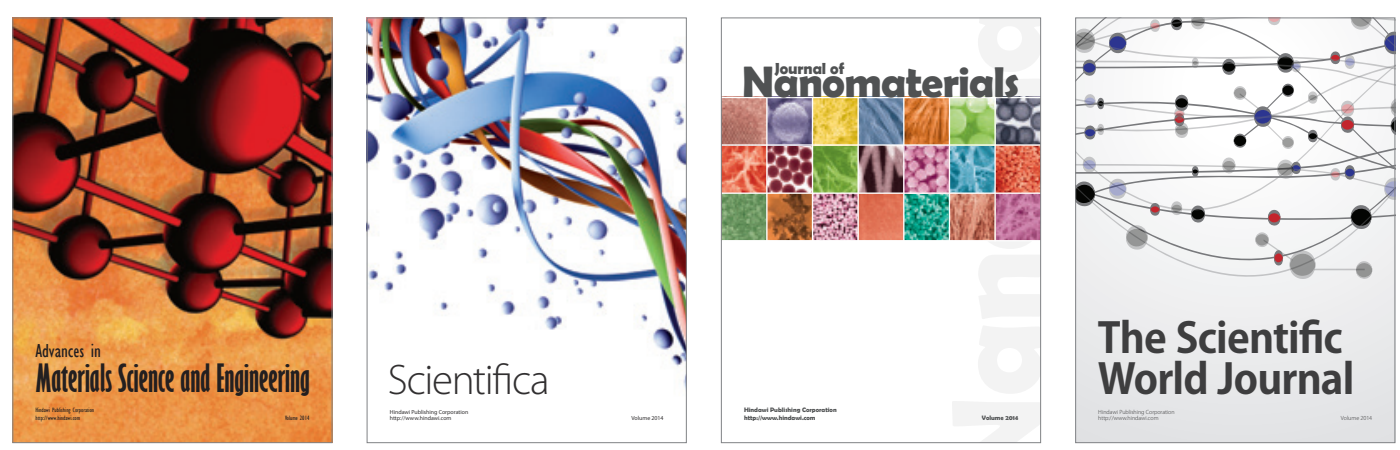

\section{The Scientific World Journal}
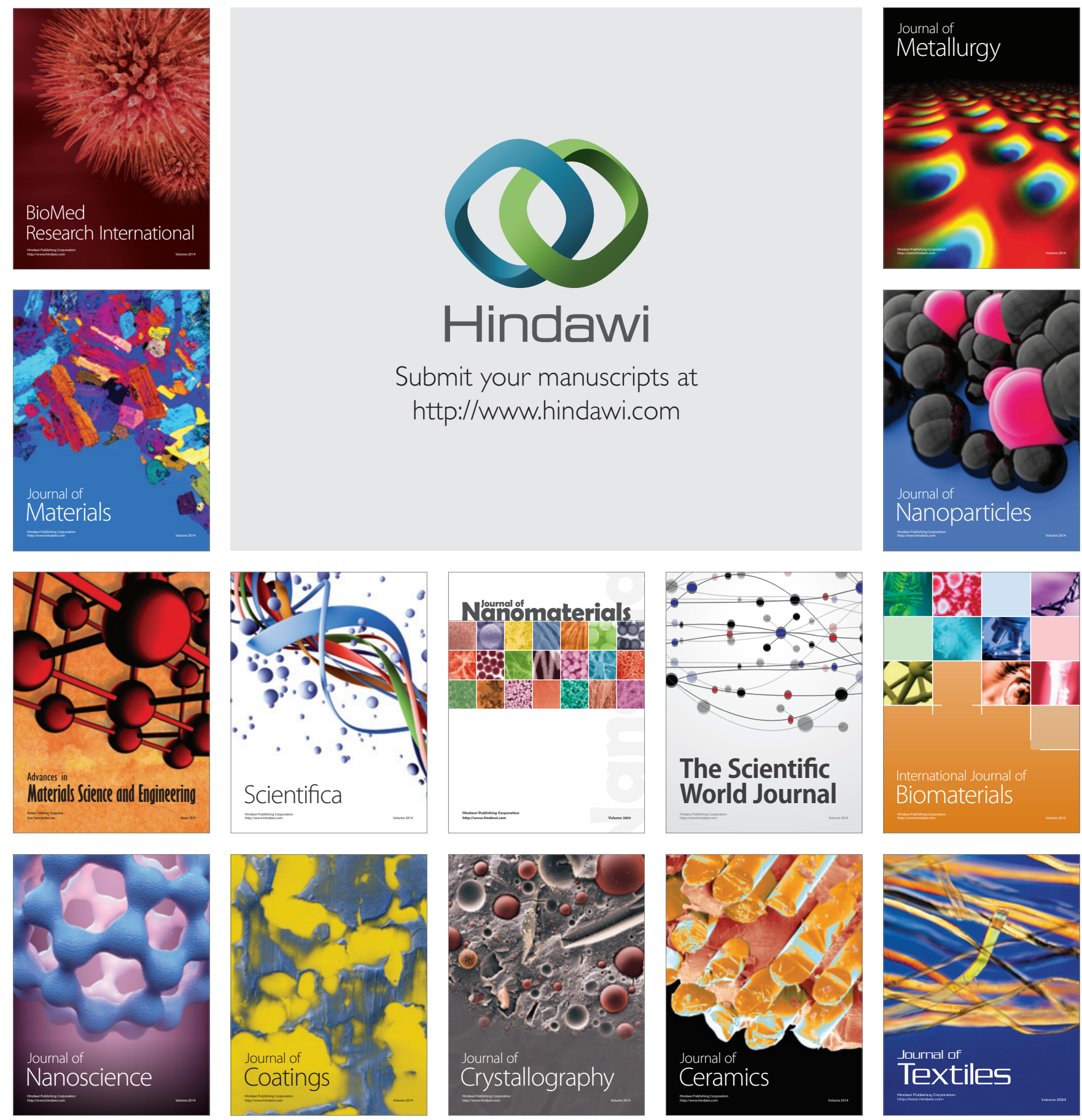